\title{
Estudio de corrientes y velocidades de aire, para la implementacion de generadores eolicos
}

\section{Study of currents and air velocities, for the implemantation of win generators}

\author{
ZAMORA-CASTRO, Sergio Aurelio*†, MOLINA-NAVARRO, Antonio, DIAZ-VEGA, María Eugenia \\ Alicia y SANTAMARIA-LOPEZ, Jesús
}

Universidad Veracruzana, Facultad de Ingenieria de la Construccion y el Habitat

ID $1^{\text {er }}$ Autor: Sergio Aurelio, Zamora-Castro / ORC ID: 0000-0002-5237-6320, CVU CONACYT ID: 104980

ID 1 ${ }^{\text {er }}$ Autor: Antonio, Molina-Navarro / ORC ID: 0000-0001-7949-8371, CVU CONACYT ID: 8011437

ID $2^{\text {do }}$ Autor: María Eugenia Alicia, Diaz-Vega / ORC ID: 0000-0002-7582-6267, CVU CONACYT ID: 351110

ID $3^{\text {er }}$ Autor: Jesús, Santamaria-Lopez / ORC ID: 0000-0003-2236-8984, CVU CONACYT ID: 404664

DOI: $10.35429 / J O E S .2019 .19 .6 .14 .18$

Recibido 09 Abril, 2019; Aceptado 30 de Junio, 2019

\section{Resumen}

La demanda de energía eléctrica y los costos de producción cada vez más elevados, aunados con la necesidad de favorecer las energías renovables, obligan a proponer soluciones alternas, que además de disminuir los costos de consumo, sean soluciones sostenibles y de muy bajo impacto ambiental. Si bien una solución se ha dado con el uso de celdas solares, éstas todavía pueden ser costosas o requerir de un mantenimiento adicional en los sistemas de fijación y soporte cuando estos se encuentran en zonas de altas concentraciones de salitre. Por lo regular estas zonas al ser costeras tienen la ventaja de recibir viento de diferentes velocidades durante prolongados espacios de tiempo durante el día, lo que puede facilitar la colocación de generadores eléctricos mediante molinos eólicos. Se realizó un estudio sobre las velocidades y direcciones del viento durante un período de tiempo, con la finalidad de establecer las condiciones y factibilidad de la colocación de estos generadores, considerando la topografía y tipología del lugar se midieron las velocidades y direcciones sobre unos de los edificios del complejo. Se obtuvo un registro que permite calcular la producción de energía y la conveniencia de explotar esta solución de energía limpia y sustentable.

Generación de energía, Sustentabilidad, Ecología

\begin{abstract}
Demand for electrical energy and rising production costs, coupled with the need to favor renewable energies, mean that alternative solutions must be proposed which, in addition to reducing consumption costs, are sustainable solutions with very low environmental impact. Although a solution has been given with the use of solar cells, these can still be expensive or require additional maintenance in the fixation and support systems when they are in zones of high nitrate concentrations. As a rule these coastal areas have the advantage of receiving wind of different speeds during prolonged periods of time during the day, which can facilitate the placement of electric generators by windmills. In the Faculty of Engineering, a zoned study was carried out on wind speeds and directions over a long period of time, in order to establish the conditions and feasibility of the placement of these generators, considering the topography and typology of the place measured speeds and directions on one of the complex buildings. Thus, a register was obtained that allows calculating the energy production and the convenience of exploiting this clean and sustainable energy solution.
\end{abstract}

Generation of energy, Sustainability, Ecology

Citación: ZAMORA-CASTRO, Sergio Aurelio, MOLINA-NAVARRO, Antonio, DIAZ-VEGA, María Eugenia Alicia y SANTAMARIALOPEZ, Jesús. Estudio de corrientes y velocidades de aire, para la implementacion de generadores eólicos. Revista de Sistemas Experimentales. 2019. 6-19: 14-18

\footnotetext{
* Correspondencia al Autor (Correo electrónico: szamora@uv.mx)

$\dagger$ Investigador contribuyendo como primer Autor
} 


\section{Introducción}

El cuidado del ambiente es una actividad primordial en nuestros días, aunado a esto la demanda en el consumo de energía cada vez es mayor y las fuentes de donde se obtuvieron regularmente generan un impacto negativo en el ambiente. Por tal motivo, se ha incursionado en los últimos años en el uso de fuentes de energía que no produzcan residuos contaminantes, o que sea mínima la producción de estos. Hoy la mayoría de la energía eléctrica que consumimos proviene de la quema de combustibles fósiles o de las presas que forman vasos que inundan grandes extensiones de lo que otrora eran variados ecosistemas.

Una alternativa más es el uso de las corrientes de aire que surcan ciertas regiones del territorio, hoy se tienen localizadas algunas zonas o corredores donde se han construido "parques eólicos", estos se conforman por una serie de molinos de grandes aspas que utilizan la fuerza que generan las corrientes de viento para mover grandes generadores eléctricos y proveer cierta zona de energía.

Sin embargo, su localización distar de las poblaciones que serán beneficiadas con la energía producida, teniéndose que construir redes de transmisión que terminan generando también cierto impacto en los ecosistemas adyacentes.

Así como en la actualidad otro medio de energía limpia como la solar, se ha podido colocar en las azoteas de las casas, el siguiente estudio pretendió analizar las corrientes del viento en una zona específica de la Ciudad de Boca del Río Ver, para determinar la factibilidad de usar generadores eléctricos cuyos molinos se muevan por el efecto de la circulación de aire a sus alrededores.

En esta investigación se realizaron los estudios y análisis a base de la observación y censo diario pertinentes para la elaboración de un Mapa de Corrientes de Aire en la zona conurbada Veracruz-Boca del Río, empleando el equipo disponible en la Facultad de Ingeniería de la Universidad Veracruzana (FIUV), además de datos proporcionados por el Centro de Previsión del Golfo perteneciente al Servicio Meteorológico Nacional, Comisión Nacional del Agua (CONAGUA).

\section{Objetivo}

Determinar mediante la obtención y análisis de los datos de las velocidades y direcciones del viento en la zona de estudio, la posibilidad de instalar generadores eléctricos a base de molinos eólicos, cuyas dimensiones sean seguras para la zona urbana y que produzcan la energía considerable para minimizar en lo posible el consumo de energía creada con combustibles fósiles y cuya inversión de operatividad sea recuperable en el corto a mediano plazo.

\section{Metodología}

La energía eólica es un recurso abundante, renovable, limpio y ayuda a disminuir las emisiones de gases de efecto invernadero al reemplazar termoeléctricas a base de combustibles fósiles, lo que la convierte en un tipo de energía verde. Sin embargo, el principal inconveniente es su intermitencia.

El uso de la energía eólica, parte de tres argumentos fundamentales:

\section{- $\quad$ El viento es limpio \\ - $\quad$ No se agota, sino que permanentemente se renueva. \\ - $\quad$ Es gratuito.}

Para poder aprovechar la energía eólica es importante hacer un estudio profuso del lugar o área designada para el montaje de todo el equipo que ha de brindar el servicio de generación de energía, en importante conocer las variaciones diurnas, nocturnas y estacionales de los vientos, la variación de la velocidad del viento con la altura sobre el suelo, la cantidad de las ráfagas en espacios de tiempo breves, y valores máximos ocurridos en series históricas de datos con una duración mínima de 20 años. (Arratia Patricio, Chocala, \& Rodriguez , 2017) Es también importante conocer la velocidad máxima del viento.

Para poder utilizar la energía del viento, es necesario que este alcance una velocidad mínima que depende del aerogenerador que se vaya a utilizar pero que suele empezar entre los $3 \mathrm{~m} / \mathrm{s}(10 \mathrm{~km} / \mathrm{h})$ y $\operatorname{los} 4 \mathrm{~m} / \mathrm{s}(14,4 \mathrm{~km} / \mathrm{h})$, velocidad llamada "cut-in speed", y que no supere $\operatorname{los} 25 \mathrm{~m} / \mathrm{s}(90 \mathrm{~km} / \mathrm{h})$, velocidad llamada "cut-out speed". (Arratia Patricio, Chocala, \& Rodriguez , 2017) 
En la actualidad México se encuentra entre los primeros lugares en generación de energía eléctrica por medio de "aerogeneradores" (Asociación Latinoamericana de Energía Eólica, 2018) por lo que es factible obtener energía y satisfacer parte de la demanda doméstica con el uso combinado de "generadores eólicos" y de "celdas solares". Específicamente refiriéndonos a la zona de estudio, la Ciudad de Boca del Río Veracruz, la posibilidad de desarrollo es indiscutible y óptima puesto que al ser una ciudad costera y rodeada de montañas da lugar a que al bajar las masas de aire éstas proporcionen un impulso a la fuerza de velocidad de los vientos, además, la superficie del mar no posee fricción contra el viento, por ende el aprovechamiento de la energía eólica generada por molinos de viento comunes, seria de excelente rendimiento energético.

Para la recolección de datos en sitio, se procedió con la selección del lugar tomando como referencia la azotea del edificio I del complejo de edificios de la Facultad de Ingeniería ubicada en Boca del Río Ver. En cuanto a la estación instalada pertenece a las series automáticas DAVI'S, el modelo es Vantage Pro2, esta puede emplearse de manera cableada o inalámbrica. Cuenta con la certificación ISO 9000. Este modelo fue elegido al presentarse la disposición para ubicarlo dentro de una oficina o lugar operativo -en este caso el laboratorio de hidráulica de la FIUV-para dejar en el interior la consola de la estación con su conexión a PC fija o portátil.

La primera fase del proyecto está encaminada a la elaboración de un mapa de corrientes mediante una base de datos de viento, esta ha de ser obtenida mediante el registro diario de la estación meteorológica DAVI'S y de los datos proporcionados por el Centro Meteorológico del Golfo, perteneciente a la Comisión Nacional del Agua CONAGUA.

Para el registro del Centro Meteorológico del Golfo ha sido necesario la utilización de cámara fotográfica para la toma de datos puesto que ahí los registros se toman de manera manual y los periodos son de cada 15 minutos, por ello el ir vaciando los datos dentro de esas instalaciones no era factible, después esos datos se han ido vaciando en la tabla de Excel previamente diseñada para cumplir con lo que se está requiriendo.
En cuanto a la información de la estación de la Facultad de ingeniería, el procedimiento fue más simplificado, las operaciones a realizar constaron en descargar la información al programa y éste se encargaba de ir generando los gráficos correspondientes mismas que explican de manera rápida las fluctuaciones de las diversas corrientes que se han presentado, si bien existe la opción de generar gráficos con la información que uno requiere, los registros de todos los sensores van almacenándose para un uso futuro; el formato en el que se presentan los datos es sencillo así pues, se procedió a homogeneizar mediante el traspaso de la información a las tablas ya diseñadas en Excel.

\section{Conclusiones}

Es necesario tomar muestras que representen el comportamiento del fenómeno que se estudia, en la investigación se tomaron los meses críticos y óptimos de acuerdo a las tablas anteriores de cada año, esto para tener una media de la cantidad de días que un aerogenerador puede funcionar en las distintas temporadas, esto despejará la duda de la continuidad de esta investigación encaminado a la instalación de un aerogenerador.

De las mediciones realizadas durante ese año, se encontró que el mes más crítico fue el de abril, ya que se registraron muchos días con velocidades del viento por debajo de la mínima necesaria para el funcionamiento de un aerogenerador. Para este mes el análisis de los resultados arroja que solo el $27.12 \%$ (Gráfica 1) del tiempo el aire sopla cuando menos con el mismo de velocidad necesaria para que un equipo pueda generar energía eléctrica

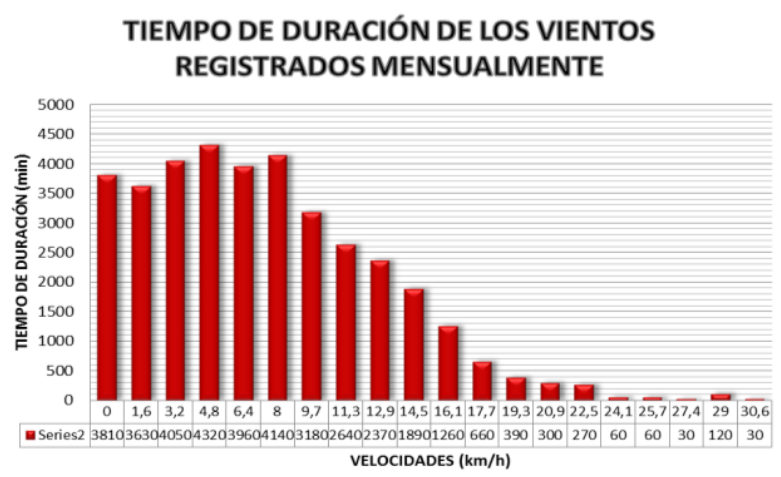

Gráfica 1 Registro de duración de vientos mensuales 


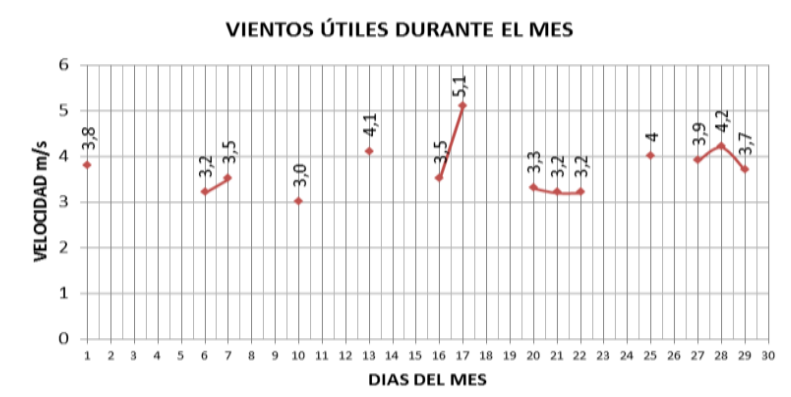

Grafica 2 Evaluación de días con velocidades a partir de $10 \mathrm{~km} / \mathrm{h}$ ó $3 \mathrm{~m} / \mathrm{s}$ mes crítico abril

En una revisión más minuciosa, se concluyó que solo siete días de ese mes eran adecuados para la generación de energía eléctrica. Sin embargo, aun cuando los días de viento útil en marzo fueron menores, solo 6 días; las velocidades fueron mucho mayores con un promedio de $35.79 \%$, por lo que la producción de energía eléctrica es mayor.

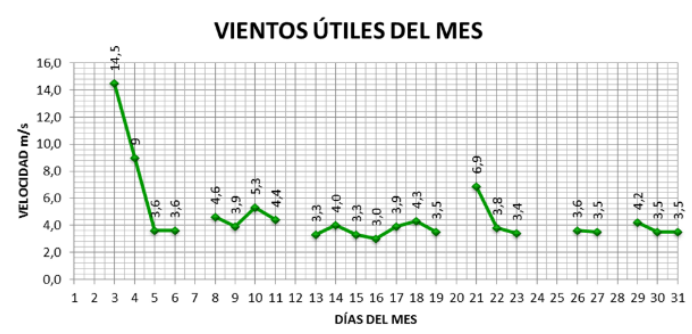

Grafica 3 Evaluación de días con velocidades a partir de $10 \mathrm{~km} / \mathrm{h}$ ó $3 \mathrm{~m} / \mathrm{s}$ mes optimo marzo

Si bien el aerogenerador deberá de adaptarse a las velocidades medias registradas, es un hecho que la continuidad de este proyecto dará los resultados esperados. Con respecto a las velocidades analizadas se determina que se mantiene una constante de $6.6 \mathrm{~m} / \mathrm{s}$, siendo que la mínima con la que un aerogenerador comercial funciona requiere de $3 \mathrm{~m} / \mathrm{s}$ o $10 \mathrm{~km} / \mathrm{h}$. Al hacer la suma de los vientos registrados por mes se ve reflejado que los meses con un registro menor de incidencia del viento ocurren en la temporada de verano, generalmente junio, julio y septiembre; por el contario los meses de mayor incremento de registro de viento se dan en febrero, marzo, noviembre y diciembre.

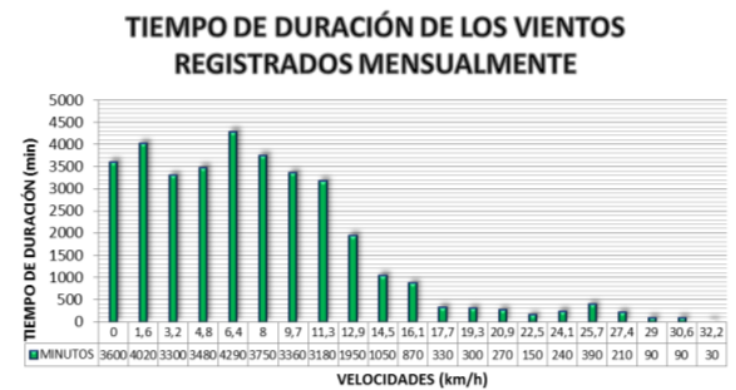

Grafica 3 Registro de duración de vientos mensuales marzo

\section{Referencias}

(Arratia Patricio, Chocala, \& Rodriguez, 2017) (Asociación Latinoamericana de Energía Eólica, 2018)

http://www.educaplus.org/climatic/01_atm_co mpo.html

http://www.oni.escuelas.edu.ar/2004/san_ju an/676/eolica_y_molino

s/capitulo_1/cap_1_1.htm

http://amdee.wsiefusion.net/amdee/que_es_la_a mdee.htm

http://www.economiadelaenergia.com/energiaeolica/

http://es.wikipedia.org/wiki/energ\%c3\%ada_e $\%$ c3\%b3lica

http://web.ing.puc.cl/ power/alumno03/alternat iva.htm

http://www.dforceblog.com/2012/02/27/co nferencia-anual-de-energia- eolica-windpower-mexico-2012/

http://www.noticiasmvs.com/noticias/intern acionales/gobierno-federal- presenta-laestrategia-nacional-de-energia-2012-2026553.html

http://www.sener.gob.mx/res/pe_y_dt/pub/ 2012/ene_2012_2026.pdf

http://www.milenio.com/topicos/milenio/energi a_eolica.aspx?o=35

http://www.milenio.com/cdb/doc/impreso/8714 185

http://es.wikipedia.org/wiki/energ\%c3\%ada_e $\%$ c3\%b3lica\#tenerg.c3 .ada_e.c3.b3lica_en_latinoam.c3.a9rica

http://www.investigacionaccion.com.ar/cate dragalan/trabajos/1b6efdf0

6b35d8f62300eeab2cb45ea2_energia_eolic a_- _informe_+_laminas_a.pdf

http://es.wikipedia.org/wiki/viento

http://es.wikipedia.org/wiki/energ\%c3\%ada_e $\%$ c3\%b3lica 
http://www.gstriatum.com/energiasolar/blog/ 2011/02/04/energia-eolica- en-mexico-2/

http://www.informador.com.mx/economia/2 011/306628/6/invertiran- 400-mdd-enparques-de-energia-eolica-en-veracruz.htm

http://es.wikipedia.org/wiki/brisa

http://portal.veracruz.gob.mx/pls/portal/docs /page/cginicio/contr aloria/planveracruzano/pemex_2008_01_1. pdf

http://www.sener.gob.mx/res/0/programa\% 20energias\%20renovables .pdf

http://www.energia.gob.mx/res/acerca_de/pr og\%20esp\%20aer.pd f

http://www.energia.gob.mx/res/pe_y_dt/pe/ mexico\%20gef\%20lsr

dp\%20pad\%207\%20june\%2020061.pdf

http://www.mercobras.com.ar/mb1lrvantage.htm

http://www.msmcomunicaciones.com/index 14 . htm

http://www.antaexclusivas.com/ant aexclusivas/dm/estacionesmeteorologicas-davis-vantage-pro2.asp? nombre $=2646 \& \operatorname{cod}=2646 \&$ s esion $=1$

http://translate.google.com.mx/translate?hl= es\&sl=en\&tl=es\&u=http $\% 3$

a\%2f\%2fwww.davisnet.com\%2fproduct_do cuments $\% 2$ fweather $\% 2$

fspec_sheets\%2f6150_spec_rev_c.pdf

http://www.portalciencia.net/meteovie.html 